Abstract 418 Table 1 Median serum levels of cytokines $(\mathrm{pg} / \mathrm{mL}$ ) in Controls and SLE patients with SLEDAI-2K $<3$ and SLEDAI-2K $\geq 3$. IQR; Interquartile range.

\begin{tabular}{|c|c|c|c|c|}
\hline & \multicolumn{3}{|c|}{ Cohort } & \multirow{2}{*}{$\begin{array}{c}\text { Independent- } \\
\text { Samples } \\
\text { Kruskal-Walis } \\
\text { test }\end{array}$} \\
\hline & Healthy Controls & SLEDAI-2KB & SLEDAI-2K $>3$ & \\
\hline cyokine & Median (IQR) & Median (IQR) & Median (IQR) & p-value \\
\hline BAFF & $980(750,1150)$ & $1590(1210,2230)$ & $1170(1320,2470)$ & $\$ 0.001$ \\
\hline$|F|-\gamma$ & $47.3(19.6,113.9)$ & $75.4(19.6,140.1)$ & $57.8(19.6,128.0)$ & 0.759 \\
\hline$\|: \cdot 1 \beta$ & $17.9(17.9,269.0)$ & $17.9(17.9,17.9)$ & $17.9(17.9,17.9)$ & 0.001 \\
\hline 111.4 & $7 \mid 7,7)$ & $7 \mid 7,7)$ & $7(7,7)$ & 0.858 \\
\hline $111 \cdot 6$ & $14(14,15.7)$ & $14(14,14)$ & $14(14,21.9)$ & 0.448 \\
\hline$\|:-10$ & $5.9(5.9,17.6)$ & $5.9(5.9,11.4)$ & $5.9(5.9,23.3)$ & 0.68 \\
\hline$\|:-12$ & $40.4(12.6,86.2)$ & $34.2(12.6,66.0)$ & $14.7(12.6,60.5)$ & 0.162 \\
\hline MCP-1 & $102.4(45.3,195.7)$ & $145.1(84.0,218.6)$ & $131.0(75.7,221.9)$ & 0.179 \\
\hline$\|:-17$ & $28.4(28.4,94.9)$ & $28.4(28.4,42)$ & $28.4(28.4,69.4)$ & 0.929 \\
\hline
\end{tabular}

not in the Principal Component 1 (PC1) of any group $(-0.22$ vs -0.18 vs 0.06$)$ (Figures 2 and 3 ).

IL-1 $\beta$ was inversely correlated with disease activity (Rs. $-0.216, p=0.013)$ and lowest in SLEDAI- $2 \mathrm{~K} \geq 3 \quad(\mathrm{p}=0.001)$. IL-1 $\beta$ was a moderate driver of cytokine variance for healthy controls, but became more dominant across SLEDAI- $2 \mathrm{~K}<3$ and SLEDAI-2K $\geq 3$, i.e. PC1 ( 0.59 vs 0.976 vs 0.985 ).

Conclusions Increased BAFF levels were not a direct agitator of cytokine variation in SLE, suggesting a contribution to disease activity through other pathways. In contrast, the reduction of IL-1 $\beta$ had a dominant effect on cytokine variance in SLE (PC 1). Principal component analysis is a useful asset for cytokine profiling in SLE.

\section{HOSPITALISATION FOR SYSTEMIC LUPUS ERYTHEMATOSUS IN WESTERN AUSTRALIA DOUBLES THE ALL-CAUSE MORTALITY RISK COMPARED TO CONTROLS, ESPECIALLY FOR MEDICARE RELIANT AND MALE PATIENTS}

${ }^{1}$ W Raymond*, ${ }^{2} \mathrm{D}$ Preen, ${ }^{1} \mathrm{H}$ Keen, ${ }^{3} \mathrm{C}$ Inderjeeth, ${ }^{1} \mathrm{~J}$ Nossent. ${ }^{1}$ The University of Western Australia, Rheumatology Group - School of Medicine, Perth, Australia; ${ }^{2}$ The University of Western Australia, School of Population Health, Perth, Australia; ${ }^{3}$ Sir Charles Gairdner and Osborne Park Hospital Group, Rheumatology, Perth, Australia
Background and aims Hospitalisation for Systemic Lupus Erythematosus (SLE) is a significant event. We aimed to understand the factors leading to an incident admission and its impact on long term outcome in SLE patients in Western Australia (WA).

Methods Using whole-population data linkage of hospital admissions, cancer registrations and death records in WA from 1980 to 2015, we performed a retrospective comparative analysis for all patients admitted with a first ever primary diagnosis of SLE (ICD-9-CM 695.4, 710.0, ICD-10-AM L93.0 and M32). For SLE patients, we analysed annual incident hospitalisation rates and compared patient characteristics, all-cause mortality and cancer risk (by Kaplan-Meier and Cox regression) versus age- and gender-matched controls free of rheumatic disease.

Results The incident hospitalisation rate for SLE (mean 20.9/ million/year (CI: 11-35) showed little variation. SLE patients were younger, more likely to be Indigenous, uninsured, have kidney and cardiovascular disorders and to die during their first hospitalisation (Table 1). Cancer risk was equivalent, but a first admission for SLE doubled the risk of subsequent death $(\mathrm{OR}=1.99$, CI: 1.5-2.7, $\mathrm{p}<0.001)$ (Figure 1, Table 2). Medicare reliance (OR 1.7, CI: 1.4-1.9, $\mathrm{p}=0.001$ ) and male gender (OR 1.4, CI: 1.0-1.8, p<0.04) were the strongest independent predictors of death. 


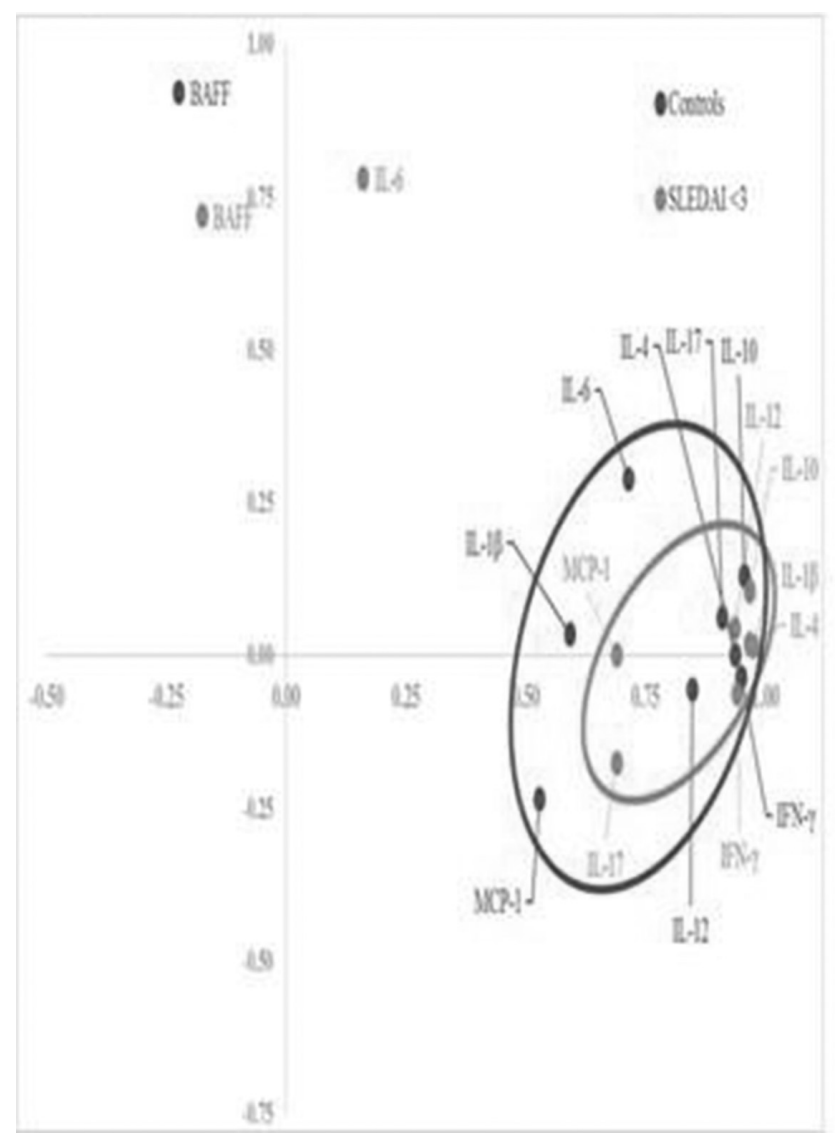

Abstract 418 Figure 2 Principal Component Analysis of Controls vs SLEDAl $<3$ group.

Conclusions Hospitalisation rates for SLE in WA have not decreased over 25 years. Once hospital-based management for SLE was required, the risk of all-cause mortality doubled compared to age and gender-matched controls. This risk was greatest in Medicare reliant or male SLE patients and not due to increased cancer risk.

\section{HOSPITALISATION FOR COMORBID CONDITIONS IN PATIENTS WITH SYSTEMIC LUPUS ERYTHEMATOSUS IS MORE FREQUENTLY DUE TO CARDIOVASCULAR AND RENAL COMPLICATIONS, SUBSEQUENTLY INCREASING THE RISK OF DEATH}

${ }^{1} \mathrm{~W}$ Raymond ${ }^{*},{ }^{2} \mathrm{D}$ Preen, ${ }^{3} \mathrm{C}$ Inderjeeth, ${ }^{4} \mathrm{H}$ Keen, ${ }^{1} \mathrm{~J}$ Nossent. ${ }^{1}$ The University of Western Australia, Rheumatology Group - School of Medicine, Perth, Australia; ${ }^{2}$ The University of Western Australia, School of Population Health, Perth, Australia; ${ }^{3}$ Sir Charles Gairdner and Osborne Park Hospital Group, Rheumatology, Perth, Australia; ${ }^{4}$ Fiona Stanley Hospital, Rheumatology, Perth, Australia

\subsection{6/lupus-2017-000215.420}

Background and aims Systemic Lupus Erythematosus (SLE) is a chronic autoimmune disease that runs an unpredictable disease course. We aimed to understand the characteristics and outcomes of incident hospitalisation for conditions other than the underlying disease in SLE patients.

Methods Using whole-population data linkage of hospital admissions and death records in Western Australia (WA) WA

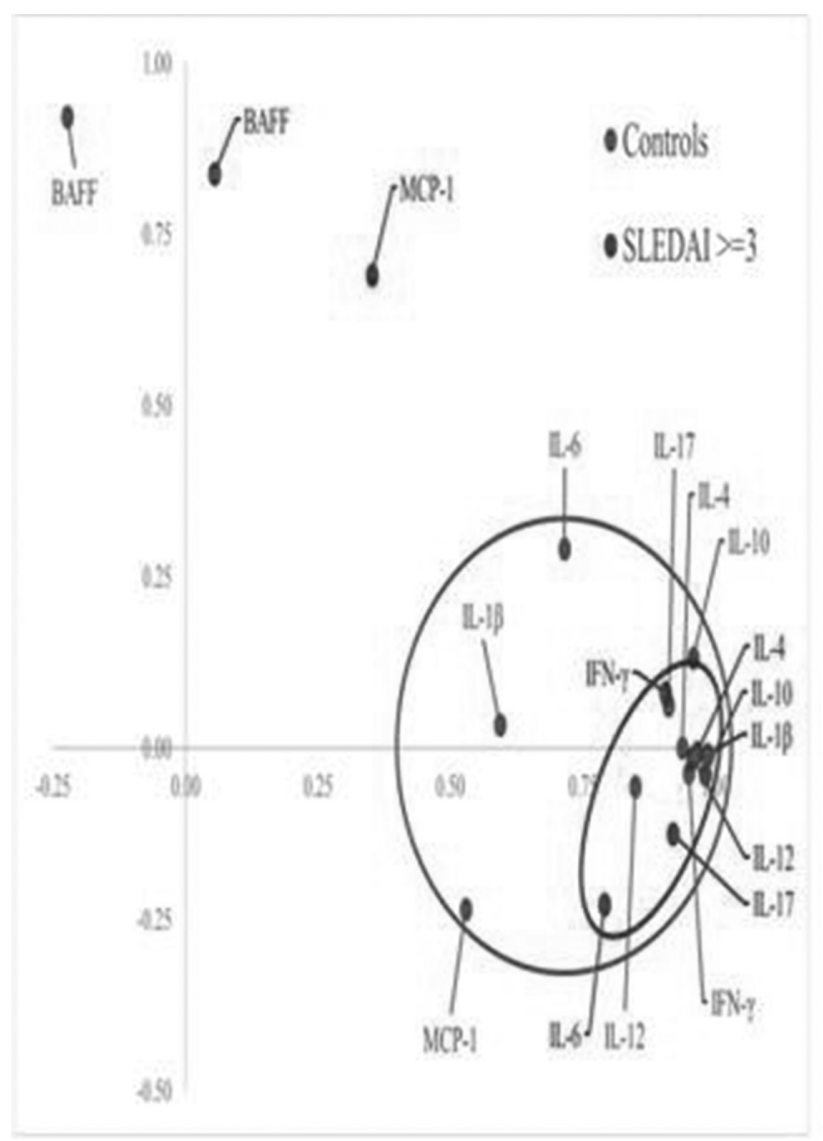

Abstract 418 Figure 3 Principal Component Analysis of Controls vs $\mathrm{SLEDAI} \geq 3$ group.

between 1980 and 2015, we performed a retrospective analysis for patients where SLE (ICD-9-CM 695.4, 710.0 and ICD10-AM L93.0 and M32) was a co-existing discharge diagnosis. All SLE patients were age- and gender-matched with hospital controls free of rheumatic disease. We investigated the rate and characteristics of the index hospitalisation for comorbidity and the risk of subsequent death by Kaplan-Meier survival and Cox regression.

Results Hospitalisation rates for comorbidity were 13.9/million/ year in SLE patients. SLE patients were similar to controls for age and gender, but more likely to be Indigenous, have renal failure, cardiovascular and thrombotic conditions (Table 1). Independent predictors of mortality risk following hospitalisation for a comorbid condition included: SLE diagnosis (OR 1.6, CI: 1.3-1.9, p<0.001) (Figure 1), cerebrovascular events (OR 2.0, CI: 1.2-3.7, p<0.001), renal disease (OR 1.75, CI: 1.4-2.3 p<0.001), thrombotic events (OR 1.8 CI: 1.1-2.8, $\mathrm{p}=0.001$ ) and reliance on Medicare (OR 1.5, CI: 1.3-1.8, $\mathrm{p}<0.001$ ) (Table 2).

Conclusions SLE patients were more frequently hospitalised than controls for cardiovascular or renal conditions and this increased their mortality risk. These results strengthen the need for close monitoring and interventions to prevent such comorbidity in all SLE patients. 